\title{
Closed-loop control of laser assisted chemical vapor deposition growth of carbon
}

nanotubes

Yoeri van de Burgt, Yves Bellouard, Rajesh Mandamparambil, Miro Haluska, and Andreas Dietzel

Citation: Journal of Applied Physics 112, 034904 (2012);

View online: https://doi.org/10.1063/1.4745874

View Table of Contents: http://aip.scitation.org/toc/jap/112/3

Published by the American Institute of Physics

\section{Articles you may be interested in}

Laser-assisted growth of carbon nanotubes-A review

Journal of Laser Applications 26, 032001 (2014); 10.2351/1.4869257

\section{Scilight}

Sharp, quick summaries illuminating the latest physics research 


\title{
Closed-loop control of laser assisted chemical vapor deposition growth of carbon nanotubes
}

\author{
Yoeri van de Burgt, ${ }^{1,2, a)}$ Yves Bellouard, ${ }^{1}$ Rajesh Mandamparambil, ${ }^{1,2}$ Miro Haluska, ${ }^{3}$ \\ and Andreas Dietzel ${ }^{1,2, b)}$ \\ ${ }^{1}$ Department of Mechanical Engineering, Eindhoven University Technology, Den Dolech 2, Eindhoven, \\ The Netherlands \\ ${ }^{2}$ Holst Centre/TNO-Netherlands Organization for Applied Scientific Research, HTC31, Eindhoven, \\ The Netherlands \\ ${ }^{3}$ Micro and Nanosystems, ETH Zurich, 8092 Zurich, Switzerland
}

(Received 20 April 2012; accepted 17 July 2012; published online 8 August 2012)

\begin{abstract}
Laser-assisted chemical vapor deposition growth is an attractive mask-less process for growing locally aligned nanotubes in selected places on temperature sensitive substrates. An essential parameter for a successful and reproducible synthesis of nanotubes is the temperature during growth. Here, we demonstrate a temperature feedback control mechanism based on the dynamic, in situ monitoring of the infrared radiation coupled with reflectivity information. With the information provided by these sensors, an infrared laser, focused on a silicon substrate covered with aluminum-oxide and iron catalyst layers, can be controlled. The growth takes place in a gaseous mixture of argon (carrier gas), hydrogen (process gas), and ethylene (carbon-containing gas). Scanning electron microscopy and Raman spectroscopy analysis demonstrate the excellent reproducibility of the closed-loop control process over multiple experiments. Furthermore, we developed a unique method to identify the onset for catalyst formation and activation by monitoring the fluctuation of the reflected laser beam. (c) 2012 American Institute of Physics. [http://dx.doi.org/10.1063/1.4745874]
\end{abstract}

\section{INTRODUCTION}

Carbon nanotubes (CNTs) are of great interest for numerous applications ${ }^{1}$ such as field effect transistors, ${ }^{2,3}$ displays, ${ }^{4}$ sensors ${ }^{5}$ flexible electronics, ${ }^{6}$ etc. CNTs are usually grown at high temperature (typically above $600^{\circ} \mathrm{C}$ ) in batch processes resulting in growth over the complete substrate. For certain applications such as interconnects, it is particularly interesting to grow CNTs only locally and without heating up the complete substrate which may contain temperature-sensitive elements.

Laser assisted chemical vapor deposition (LACVD) offers an attractive method for the local growth of CNTs. Previous studies have demonstrated the possibility of growing different types of carbon nanotubes such as, singlewalled $^{7}$ or multi-walled, ${ }^{8}$ with different laser types ${ }^{9}$ and catalysts. ${ }^{10}$ Temperature monitoring and control of laser assisted growth have been demonstrated before by using an homogeneous substrate temperature ${ }^{7}$ or large spot sizes, ${ }^{11}$ demanding very high laser power.

Apart from the temperature, an important parameter for CVD processes that determines the CNT properties and diameter is the catalyst nano-particles and their preparation process. $^{12,13}$ It is generally assumed that the catalyst layer first is oxidized $^{14}$ in air and then has to be reduced by a heat treatment in the presence of hydrogen ${ }^{13}$ to finally form nanoparticles. ${ }^{12,15}$ In the case of a laser CVD process, the sequence of events and

\footnotetext{
a) Tel.: +31402472186. E-mail: y.b.v.d.burgt@tue.nl.

${ }^{b)}$ Present address: Institute of Microtechnology (IMT), TU Braunschweig, Langer Kamp 8, 38106 Braunschweig, Germany.
}

their dynamics leading to the formation of nanoparticles is barely understood. This step is nevertheless crucial for controlling the growth product and its characteristics.

In this research, we demonstrate a feedback control mechanism for a laser assisted CVD process, based on monitoring the mid-infrared radiation from the laser affected zone. This radiation is essentially of thermal origin and provides indirect information about the average temperature at the irradiated spot. To monitor surface changes during laser exposure as well as to detect the onset for CNT growth, we monitor the reflected laser intensity. Indeed, we have shown in a previous work ${ }^{16}$ that this signal can be correlated to various critical steps of the CNT growth. Here, we show that this signal can be further used for identifying the catalyst formation and their activation to catalyze the carbonaceous gas dissociation and CNT nucleation. To quantitatively relate the temperature to its influence on the quality of the CNTs, a finite element model that takes into account time-dependent laser absorbed intensity is developed.

We first describe the experimental setup and the control strategy. In a second part, we discuss the thermal model that we fit with experimental data. Then, we investigate the catalyst nanoparticle formation and finally, we conclude with presenting experimental results that demonstrate the usefulness and the necessity of a closed-loop control.

\section{EXPERIMENTAL}

\section{A. Experimental setup}

The setup is shown schematically in Fig. 1. The substrates are $4 \times 4 \mathrm{~mm}$ silicon substrates with a $20 \mathrm{~nm}$ 


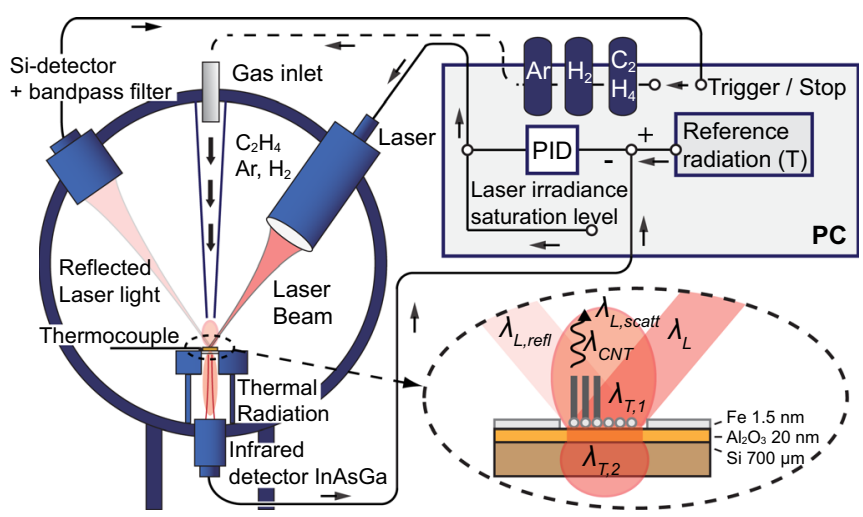

FIG. 1. Overview of the laser assisted chemical vapor deposition setup with feedback control. The flow of operation is depicted by arrows. The set process parameters are laser irradiance saturation level and the reference radiation. The thermal radiation from the substrate is controlled by means of a feedback signal from the infrared detector. The reflected laser light is collected by a Si-detector with a bandpass-filter to isolate the laser wavelength. The inset illustrates the formation of the nanoparticles (right) on the catalyst followed by the CNT growth (left) on the substrate during laser irradiation. The different wavelengths of radiation present in the system are shown. The incoming laser light is $\lambda_{L}(808 \mathrm{~nm}), \lambda_{L \text { reft }}$ is the reflected laser light, $\lambda_{L \text { scatt }}$ is the scattered laser light, $\lambda_{C N T}$ is the fluorescence of the CNTs during growth, and $\lambda_{T, 1}$ and $\lambda_{T, 2}$ are the emitted thermal radiation from the spot.

aluminum-oxide layer and a $1.5 \mathrm{~nm}$ iron catalyst layer both deposited by e-beam evaporation. The stainless steel cylindrical chamber has a volume of 3.41 with a gas inlet and an outlet. A K-type thermocouple is placed at the substrate holder, a pressure sensor is placed in the gas inlet, and an optical window provides visual access. A fiber-pigtailed laser diode (unique mode) emitting up to $35 \mathrm{~W}$ at $\lambda_{L}=808 \mathrm{~nm}$ is incident at an angle of $35^{\circ}$. An InGaAs photodiode is placed beneath the substrate to measure the emitted IR radiation. In this configuration, the Si substrate acts as a high-pass optical filter with a cutoff wavelength of $1 \mu \mathrm{m}$. The reflected laser light is measured with a Si photodiode in combination with a laser line band-pass optical filter. The chamber is filled with a mixture of argon, hydrogen, and ethylene to $950 \mathrm{mbar}$ in the ratio $8 / 2 / 5$, respectively. The CNTs are grown in a controlled way and the results are analyzed using a scanning electron microscope (FEI Quanta 600F ESEM) and a Raman spectrometer (632 nm, Horiba LabRAM HR). The thermal finite element model is developed in COMSOL MULTIPHYSICS using the heat transfer Computational Fluid Dynamics (CFD) module with a time-dependent Generalized minimal residual method (GMRES) solver.

\section{B. Control strategy}

Open-loop laser assisted CVD is intrinsically sensitive to perturbations due to the small size of the heated zone but also to rapid changes of surface properties that modify the laser absorption and in turn the temperature. In Fig. 2, a schematic of the open loop system is presented in the top right blue area. In open-loop, the laser irradiance determines solely the process temperature and the rate at which the temperature increases $(\mathrm{dT} / \mathrm{dt})$. During the growth of CNTs, the absorbance of the substrate changes resulting in a different thermal behavior and ultimately a different temperature.

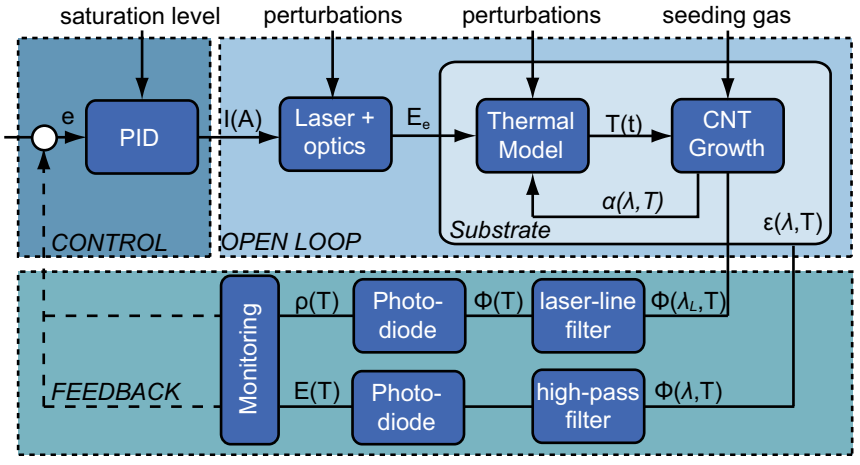

FIG. 2. Schematic of the laser assisted carbon nanotube growth process and all the relevant components. In open-loop, a set current is converted into certain laser energy $\mathrm{E}_{\mathrm{e}}$ which is irradiated on the substrate. The thermal model of the substrate transforms this energy into a temperature $\mathrm{T}$ at which the CNT growth starts. This growth and the temperature increase change the absorbance $\alpha(\lambda, T)$ of the substrate which in turn re-affects the thermal model. Along the open-loop process line perturbations are present. The reflected laser flux $\Phi\left(\lambda_{\mathrm{L}}, \mathrm{T}\right)$ is depending on the absorbance $\alpha(\lambda, \mathrm{T})$ of the substrate and is measured by a Si-photodiode. The emissivity $\varepsilon(\lambda, \mathrm{T})$ of the system transforms the temperature of the laser spot and surroundings into a flux $\Phi(\lambda, T)$. This flux is measured by an InGaAs photodiode after passing through the silicon substrate which acts as a high-pass filter. A PID controller with a set saturation level is used to close the loop.

Extreme situations such as overheating can occur, preventing the CNTs from growing further.

To counteract perturbations and to better control the growth process, a closed-loop model is developed as depicted in the bottom section of Fig. 2. The reflected laser beam and the emitted thermal radiation from the irradiated spot on the substrate are given as inputs for the closed-loop control. The strategy is to monitor and control the emitted radiation $\varepsilon(\lambda, \mathrm{T})$ from the laser spot which is directly related to the temperature. The reflectance of the laser heated surface $\rho\left(\lambda_{\mathrm{L}}, \mathrm{T}\right)$ is assumed to be the direct complement of the absorbance $\alpha\left(\lambda_{\mathrm{L}}, \mathrm{T}\right)$ of the substrate (in other words, the transmitted laser energy through the substrate can be neglected). As a consequence, a decrease in reflectance results in more absorbance of the laser irradiation and therefore an increased heating.

As shown in Fig. 2, a proportional-integral-derivative (PID) controller is used for the feedback process. For a step response to a given reference radiation, the initial laser irradiance will be equal to the selected laser irradiance saturation level. This level determines the maximum value the controller will achieve and ensures that no integrator windup can occur. This phenomenon could otherwise occur when the output is limited (i.e., a maximum laser diode output) but the reference set-point is not yet reached. In this configuration, it therefore also limits the maximum irradiance output for the laser diode. In contrast to the open-loop situation, at the onset of the closed-loop process, the laser saturation irradiance determines the heating rate during the start of the process but does not determine the temperature at the laser spot, since the irradiance is controlled to keep the radiation constant during the continuation of the process.

In Fig. 1, the sensors are shown in their actual configuration in the setup. The flow of operation is depicted by arrows. The inset of the figure shows a detailed schematic of the 
different radiated, reflected, scattered, and emitted wavelengths involved in the process. The incoming laser light is $\lambda_{L}(808 \mathrm{~nm}), \lambda_{\text {L refl }}$ is the reflected laser light after hitting the surface of the substrate, $\lambda_{L \text { scatt }}$ is the scattered laser light, $\lambda_{C N T}$ is the fluorescence emitted by the CNTs during growth, and $\lambda_{T, 1}$ and $\lambda_{T, 2}$ are spectra of emitted thermal radiation from the spot. The light emitted by the CNTs is currently not measured dynamically and therefore not taken into account in the closed-loop control process.

\section{RESULTS AND DISCUSSION}

\section{A. Thermal model}

One of the difficulties of laser assisted growth of CNTs is to accurately predict the temperature at the laser spot. As shown in Fig. 2, the thermal model is an important element of the control process. Since the growth process is mainly dependent on temperature evolution in time, it is desirable to understand and adapt the growth process in relation to that. Finite element analysis software (COMSOL) is used to implement the thermal model of the process to be able to calculate the temperature evolution during the growth process. A 3D sketch of the model is shown in Fig. 3. The substrate holder is an aluminum cylinder with a hole in the middle covered by a fused silica slide. The silicon substrate is fixed by two iron springs on top of the fused silica. The substrate holder is itself placed on top of a steel cylindrical part of the chamber. The inside of the cylinders is hollow so that the emitted radiation can be collected beneath the substrate. In the model, the temperature is calculated for each time step using the relevant heat conduction, radiation, and convection of different materials and gasses in the system.

The temperatures at the spot and surrounding are in the order of $600-1100 \mathrm{~K}$ so the thermal conductivity and heat capacity of silicon, fused silica, and iron are given temperature-dependent values. In order to incorporate convection, the temperature-dependent thermal conductivity, kinematic viscosity, and thermal diffusivity of the surrounding gases (argon, ethylene, and hydrogen ${ }^{17}$ ) are used to calculate

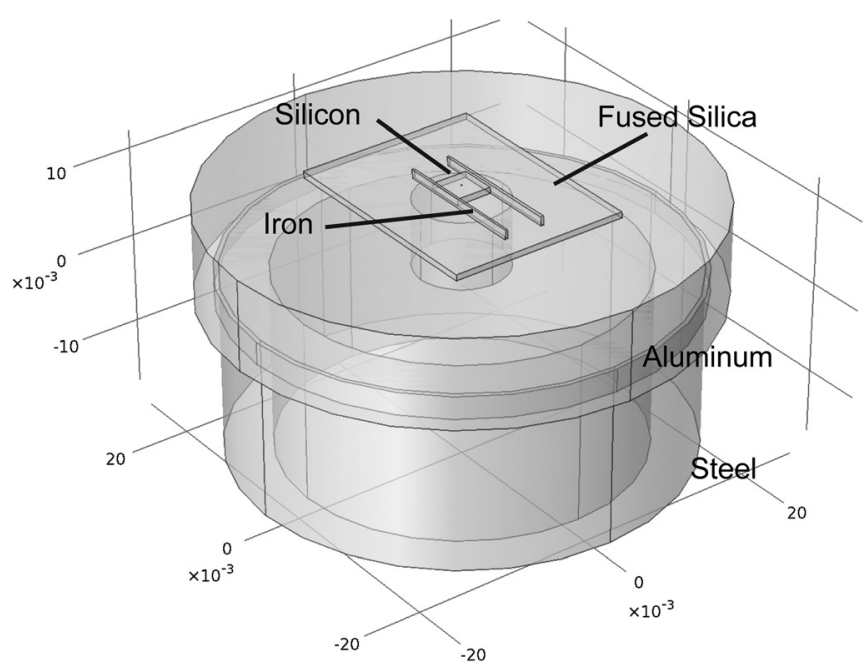

FIG. 3. Sketch of the setup used in the finite element model in COMSOL. In the picture, the different materials are labeled. the temperature-dependent heat transfer coefficient $h_{c}$ at different surfaces. To estimate these coefficients, the following Nusselt numbers for free convection from a horizontal-top, bottom and a vertical ${ }^{18}$ plate respectively are used

$$
\begin{gathered}
\mathrm{Nu}_{H T}=0.54 \mathrm{Ra}^{1 / 4}, \\
\mathrm{Nu}_{H B}=0.27 \mathrm{Ra}^{1 / 4} \\
\mathrm{Nu}_{V P}=0.68+\frac{0.67 \mathrm{Ra}^{1 / 4}}{\left(1+(0.492 / \mathrm{Pr})^{9 / 16}\right)^{4 / 9}}
\end{gathered}
$$

where Ra and Pr are the Rayleigh and the Prandtl numbers. The Nusselt number is defined as the ratio between the convective and conductive heat transfers. The heat transfer coefficient is given by,

$$
h_{c}=\mathrm{Nu} \times k_{f} / L,
$$

where $k_{f}$ is the thermal conductivity of the gas mixture and L is the characteristic length or diameter. The laser spot is modeled as an ellipsoid with dimensions $65 \times 80 \mu \mathrm{m}$ to take into account the incidence angle of $35^{\circ}$. The beam has a Gaussian intensity profile.

To compare the model with reality, three different experiments are performed in an argon/hydrogen environment to ensure the absence of ethylene where no growth can take place. In Fig. 4, the temperature evolution of these experiments using different laser irradiances is shown and compared with the experimental data from the thermocouple positioned at the edge of the substrate.

\section{B. Catalyst}

The formation of nano-particles from the catalyst layer is a crucial process since this determines the properties and characteristics of the carbon nanotubes. To investigate the formation of the catalyst nano-particles, the substrates used in the open-loop experiments of Fig. 4 are observed since they do not contain any carbonaceous growth. In Fig. 5, the

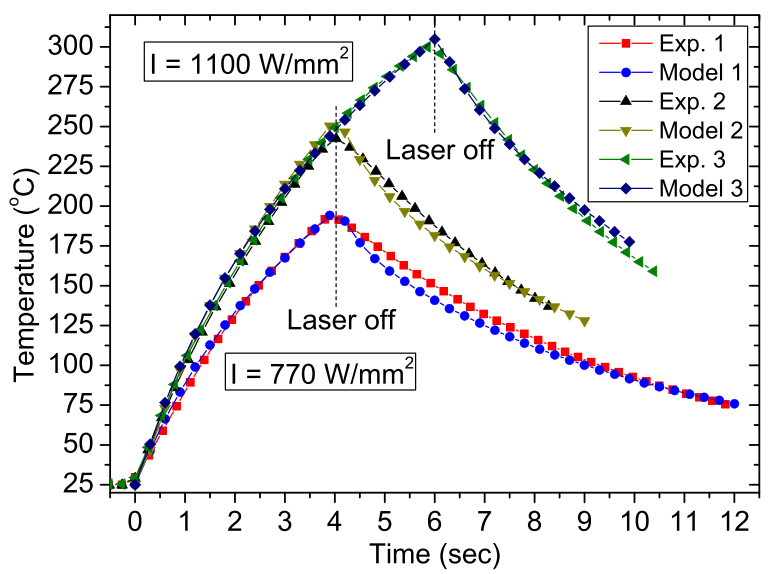

FIG. 4. Plot showing the comparison of the temperature at the edge of the silicon substrate from the thermal model with experimental data for different laser irradiances. Two different laser irradiances and two different switch off times are presented. All experiments are performed in open-loop configuration. 


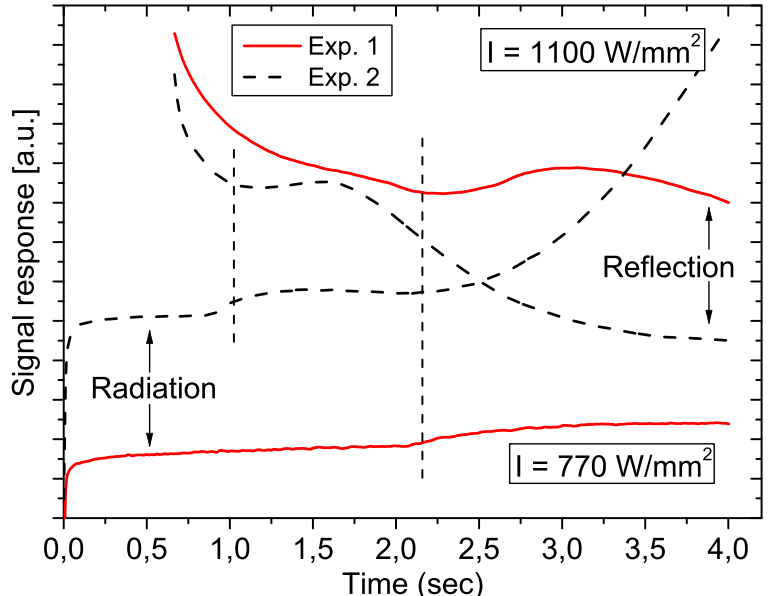

FIG. 5. Detector response for experiments 1 (solid red) and 2 (black dashed). The emitted radiation of experiment 1 is lower which is due to the lower laser irradiance. For the radiation of experiments 1 and 2, an increase is visible around 1 and $2 \mathrm{~s}$, respectively (see dashed lines). At the same time, a small "step" in the reflection is visible. This increase is attributed to the formation and reduction of the iron catalyst nano-particles.

radiation and reflection detector responses for experiments 1 and 2 are presented. The radiation is lower for experiment 1 due to the lower laser irradiance. For both experiments, an increase of the radiation signal was observed. This increase in thermal radiation is attributed to the reduction and formation of the catalyst nano-particles. The $1.5 \mathrm{~nm}$-thick iron catalyst layer is fully oxidized prior to starting the process. ${ }^{14}$ The absorbed energy is used to reduce with hydrogen and melt the oxidized iron layer that turns into small metallic nanoparticles on top of the $\mathrm{Al}_{2} \mathrm{O}_{3}$ layer. The higher the laser irradiance, the faster this process is completed. This is confirmed by the reflected laser signal in Fig. 5 .

In Fig. 6, a schematic overview of the reflected laser signal over time and the proposed mechanism corresponding to the different signal intensities is given. Inset (a) illustrates the starting configuration. The reflection first drops (inset (b)) due to the increase in absorbance of the laser irradiance

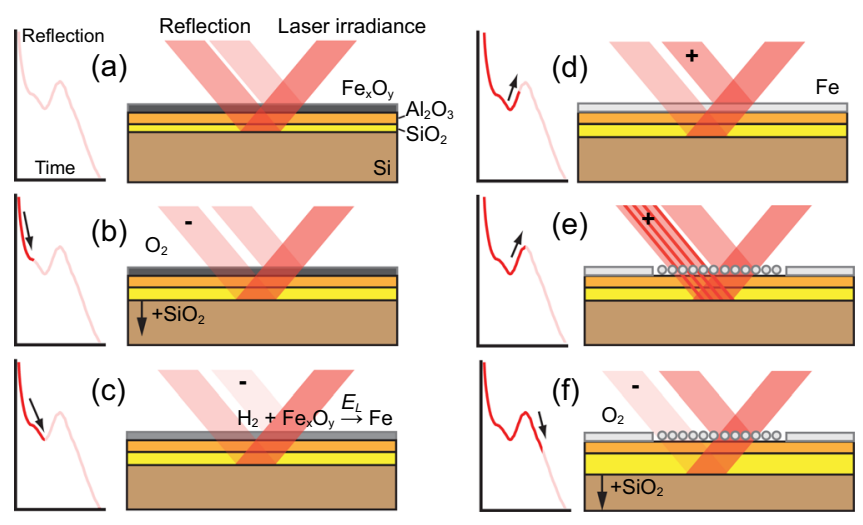

FIG. 6. Schematic of the catalyst nano-particle formation process. (a) Starting configuration. (b) Reflection is decreased due to enhanced absorbance as a result of temperature increase. (c) Reflection is decreased due to the absorbance of energy for the reduction process. (d) Reflection is increased due to the formation of the metallic layer. (e) Reflection is increased due to the enhanced passing of the reflection from the layers beneath the catalyst as a result of the nanoparticle formation. (f) Reflection is decreased by further enhanced absorbance and interference effects as a result of the growth of $\mathrm{SiO}_{2}$.
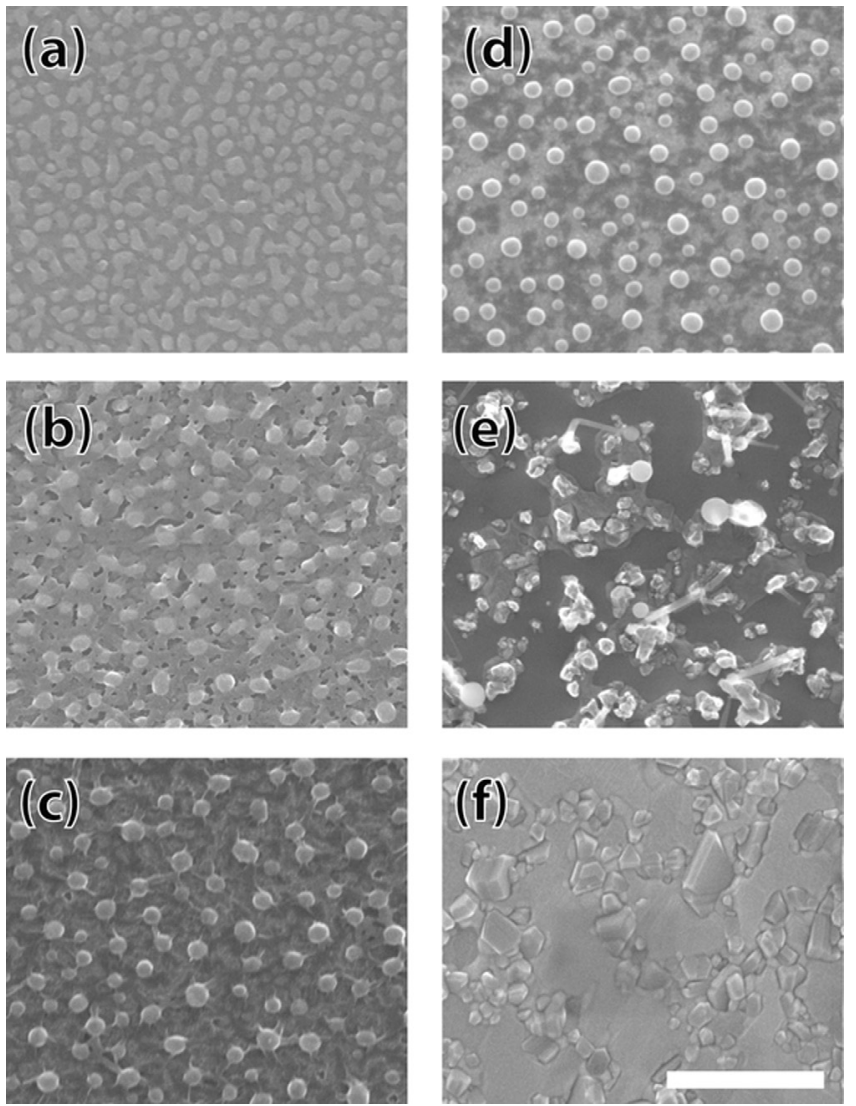

FIG. 7. Scanning electron microscopy pictures of the laser affected zone of the catalyst layer after different exposure irradiances (I) and times (t): (a) $\mathrm{I}=7.7 \times 10^{8} \mathrm{~W} / \mathrm{m}^{2}$ and $\mathrm{t}=4 \mathrm{~s}$. (b) $\mathrm{I}=9.4 \times 10^{8} \mathrm{~W} / \mathrm{m}^{2}$ and $\mathrm{t}=4 \mathrm{~s}$. (c) $\mathrm{I}=1.1 \times 10^{9} \mathrm{~W} / \mathrm{m}^{2}$ and $\mathrm{t}=4 \mathrm{~s}$. (d) $\mathrm{I}=1.1 \times 10^{9} \mathrm{~W} / \mathrm{m}^{2}$ and $\mathrm{t}=6 \mathrm{~s}$. (e) $\mathrm{I}=1.1 \times 10^{9} \mathrm{~W} / \mathrm{m}^{2}$ and $\mathrm{t}=10 \mathrm{~s}$. (f) $\mathrm{I}=1.1 \times 10^{9} \mathrm{~W} / \mathrm{m}^{2}$ and $\mathrm{t}=40 \mathrm{~s}$. The scale bar represents $500 \mathrm{~nm}$.

as a result of the temperature increase. The reduction into metallic iron and the melting of the layer consume energy which results in a further drop in reflection (c). However, the metallic iron layer increases the reflection of that layer (d). The formation of small nano-particles reduces the actual covered area of the layer which also contributes to an increase in reflection (e). The heating of the substrate further decreases the reflectance due to the enhanced absorbance and possible additional interference effects associated with the thermal growth of $\mathrm{SiO}_{2}$ (f). This peak in intensity takes longer for the lower laser irradiance in Fig. 5 to occur and corresponds in time to the increase in radiation. A higher laser irradiance generally leads to smaller catalyst particles and ultimately to nanotubes with a smaller diameter. ${ }^{15,19,20}$ However, an overly long laser exposure will lead to the destruction of the catalyst layer and the formation of oxides. In Fig. 7, the scanning electron microscopy (SEM) pictures of the catalyst layer are shown for different laser irradiances and exposure time. It is observed that at higher laser irradiance and longer exposure time more discrete separation of the catalyst nano-particles occurs. This can be seen in Figs. 7(a)-7(d). However, 7(e) and 7(f) show a destruction of the catalyst layer and the formation of oxide crystals.

To demonstrate that the peak in the reflection signal is a result of the formation and reduction of the catalyst, we compare the reflected laser signal from three experiments, two 


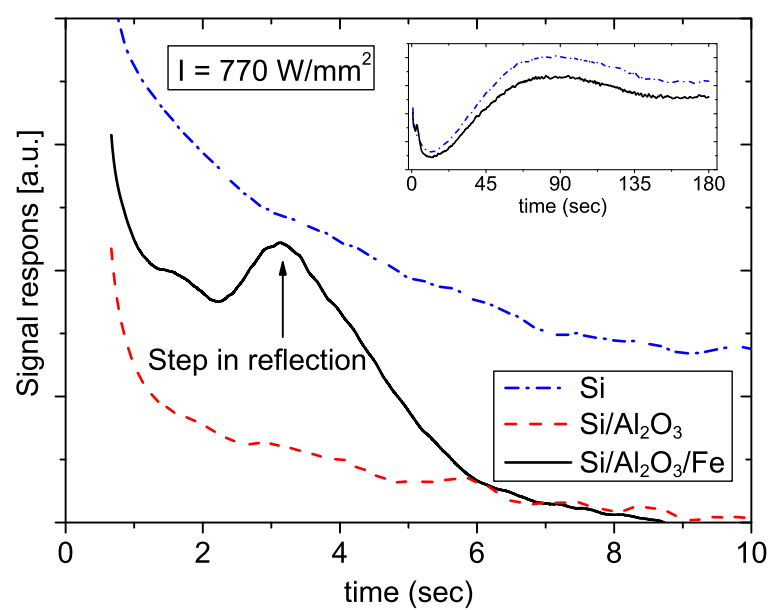

FIG. 8. Reflected laser irradiance response for three different cases: (1) a silicon substrate with only native oxide, (2) a silicon substrate with only $20 \mathrm{~nm}$ $\mathrm{Al}_{2} \mathrm{O}_{3}$, and (3) the conventional substrate, a silicon substrate with $20 \mathrm{~nm}$ $\mathrm{Al}_{2} \mathrm{O}_{3}$ and $1.5 \mathrm{~nm}$ iron. It is clear to see that the hill in the reflection only occurs when the iron layer is present and is therefore attributed to the formation of the catalyst particles.

substrates without the iron catalyst and one with the catalyst layer. These results are shown in Fig. 8. As seen in this figure, the peak only occurs when the iron layer is present. When the substrate with the iron layer is exposed again to the same laser exposure conditions, the peak in the reflection is not observed, indicating that the formation and reduction of the iron catalyst nano-particles already took place. At this stage and due to the lack of experimental data for various catalyst thicknesses, we cannot conclude a possible correlation between the amplitude of the peak and the size of the nanoparticles. The inset of the figure shows an extension of the reflected laser intensity for $3 \mathrm{~min}$. Since both curves ( $\mathrm{Si}$ and $\mathrm{Si} / \mathrm{Al}_{2} \mathrm{O}_{3} / \mathrm{Fe}$ ) are showing an equal oscillation pattern, we attribute these oscillations to an optical interference effect caused by the thermal growth of $\mathrm{a} \mathrm{SiO}_{2}$ layer, as proposed in Fig 6, since that is the only material present on both substrates.

To further confirm this assumption, we investigate the influence of the surrounding process gasses. Previous research has already indicated the importance of hydrogen in the formation and reduction (de-oxidation) of the iron catalyst nano-particles. ${ }^{13}$ It is generally believed that the thin iron layer is quickly oxidized after being exposed to air into a combination of magnetite $\left(\mathrm{Fe}_{3} \mathrm{O}_{4}\right)$ and maghemite $(\gamma$ $\mathrm{Fe}_{2} \mathrm{O}_{3}$ ), a form of hematite. ${ }^{14}$ The heat from the laser in combination with the hydrogen reduces this iron-oxide back to iron using the following redox relations: ${ }^{21,22}$

$$
\begin{gathered}
3 \mathrm{Fe}_{2} \mathrm{O}_{3}+\mathrm{H}_{2} \longrightarrow 2 \mathrm{Fe}_{3} \mathrm{O}_{4}+\mathrm{H}_{2} \mathrm{O} \\
\mathrm{Fe}_{3} \mathrm{O}_{4}+4 \mathrm{H}_{2} \longrightarrow 3 \mathrm{Fe}+4 \mathrm{H}_{2} \mathrm{O} .
\end{gathered}
$$

The high temperature melts the iron and surface energy minimization of the iron liquid on the alumina surface causes the formation of small nano-particles as a result of the strong catalyst-support interaction. $^{23}$ Note that the nanoparticles' size, mobility, and distribution are also dependent on the method of deposition of both the support and the catalyst. ${ }^{24}$

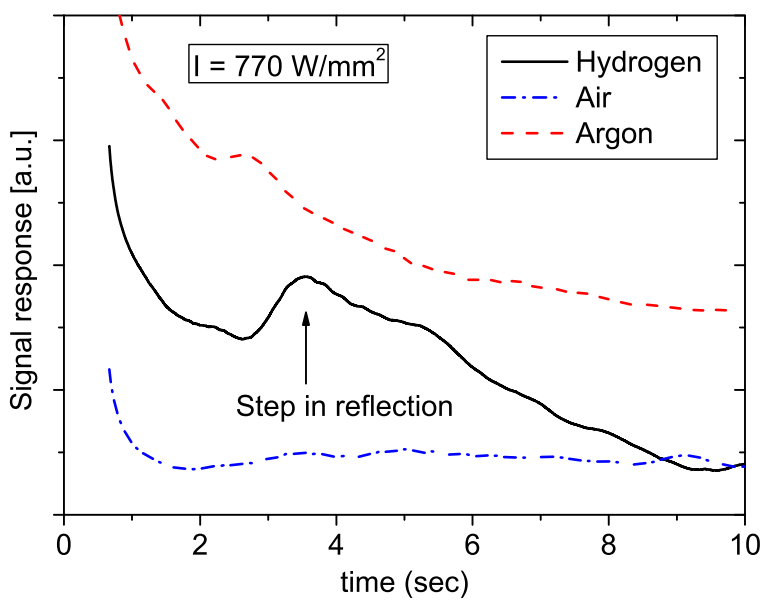

FIG. 9. Reflected laser irradiance response for a silicon $/ \mathrm{Al}_{2} \mathrm{O}_{3} / \mathrm{Fe}$ substrate in different environments: hydrogen, argon, or air, with the same laser irradiation. Only the hydrogen case shows a step in the signal, which indicates a reduction process and is therefore attributed to the formation and reduction of the $\mathrm{Fe}_{2} \mathrm{O}_{3}$ layer into $\mathrm{Fe}$ nanoparticles.

At a temperature above $577^{\circ} \mathrm{C}$, wüstite $(\mathrm{FeO})$ can nucleate. This oxide is more stable and harder to reduce. ${ }^{25}$ To avoid the nucleation of this oxide, the time that the process exceeds this temperature in the absence of CNT growth should be minimized. Equations (5) and (6) indicated that the formation of iron nano-particles will not occur in the absence of hydrogen. In Fig. 9, the laser reflection is shown for a substrate with the iron catalyst in the presence of different gases. When only air or argon is present, there is no observable peak in the reflection signal. When only hydrogen is present in the reaction chamber, there is a prominent peak present in the reflection. This experimentally confirms that the peak in the reflection signal is a result of the reduction and formation of the iron catalyst nano-particles. Our in situ catalyst observations demonstrate that the reflected signal can be used to monitor the onset of the catalyst layer activation. This important information can be used to trigger the growth process by introducing the ethylene at that time.

\section{Temperature closed-loop control based on IR radiation}

Using the time dependent laser irradiance and reflected laser signal, we use the finite element model to calculate the temperature as a function of time for experiments in the closed-loop configuration as described before. In Fig. 10, the reference radiation, measured in voltage, is compared with the modeled maximum temperature calculated from different experiments. The figure demonstrates the direct relation between the reference radiation set-point and the process temperature at the laser spot. The inset of the figure shows the relation between the selected saturation laser irradiance level and the calculated temperature for a constant reference set-point (in this case $0.4 \mathrm{~V}$ ) for the radiation signal. It shows that there is no visible influence on the temperature at the growth site for increasing laser irradiance saturation level.

We have also shown in the previous work $^{16}$ that in open-loop the laser set-point, as one would expect, governs the rate of temperature increase and is related to the quality 


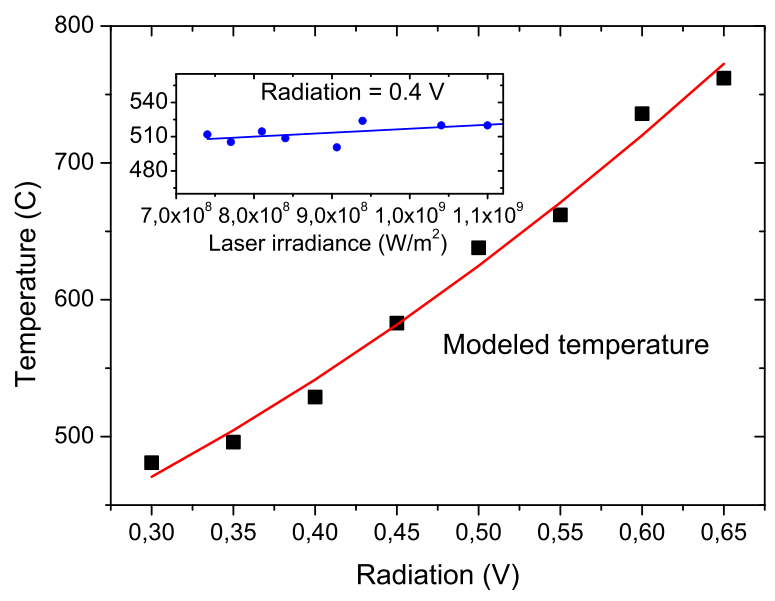

FIG. 10. Plot of the modeled peak temperature vs the set-point reference radiation measured as voltage from the InGaAs detector. Plotted are the maximum peak values at the laser spot for a laser saturation irradiance of $1100 \mathrm{~W} / \mathrm{mm}^{2}$. The inset shows the temperature as a function of selected saturation laser irradiance with the reference radiation set at $0.4 \mathrm{~V}$.

of the growth product. A high temperature increase rate-in other words, a fast heating-yields higher quality CNTs. In the closed-loop situation, a higher laser irradiance saturation level would mean a faster response to the set-point value, which is analogue to achieving the CNT growth temperature faster. This can be seen in Fig. 11 for two different laser irradiances. For a laser saturation irradiance of $1100 \mathrm{~W} / \mathrm{mm}^{2}$, the set point is reached faster than in the case of $770 \mathrm{~W} / \mathrm{mm}^{2}$. A too fast response (i.e., too high laser irradiance) can lead to an overshoot in the set-point (or would require adapting the PID coefficients). In Fig. 11 also the difference between the temperature at the laser spot for the controlled and uncontrolled situations is depicted. Both experiments start with a saturation laser irradiance level of $770 \mathrm{~W} / \mathrm{mm}^{2}$. The laser irradiance of the controlled experiment, on the other hand, drops in time to keep the radiation at the constant reference value. Until around $4 \mathrm{~s}$, the temperature increase is more or less equal for both situations. The small dip is attributed to the formation and reduction of the iron catalyst nanoparticles.

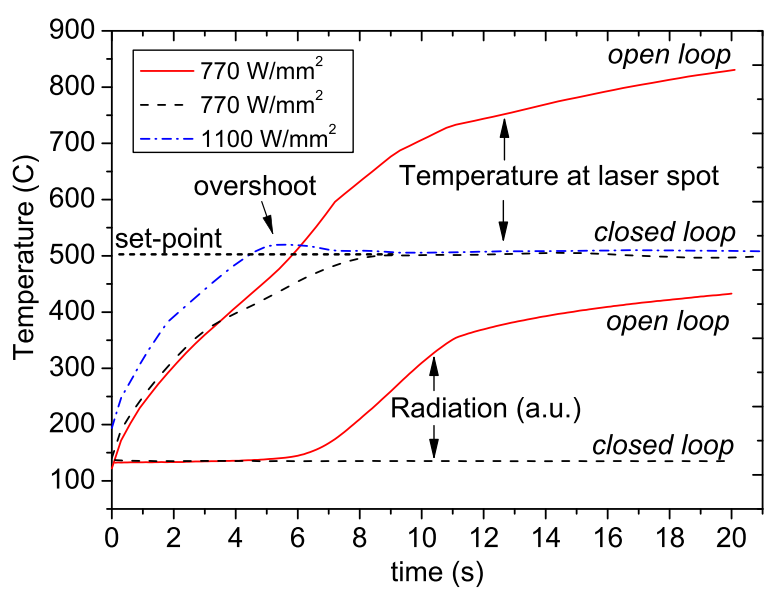

FIG. 11. Temperature at the laser spot as a function of time for the uncontrolled (black dashed) and radiation-controlled situation (red solid and blue dash dotted). The set-point is shown with an overshoot. The radiation is also plotted for both situations.
The quality of the CNTs is characterized by using Raman spectroscopy. ${ }^{26-28}$ The ratio of the D-band to G-band spectral intensity can be used as a measure for the quality of the nanotubes in terms of the presence of structural defects on nanotube walls and of amorphous carbon as well. High quality nanotubes with a low concentration of structural defects and amorphous carbon deposit in general have a low $\mathrm{I}(\mathrm{D}) / \mathrm{I}(\mathrm{G})$ ratio. The $\mathrm{G}^{\prime}$-band occurs around $2650 \mathrm{~cm}^{-1}$ and corresponds to the overtone of the D-band. The $\mathrm{G}^{\prime}$-band originates from a double resonance process involving two phonons from the corners of the first Brillouin zone. ${ }^{26}$ In contrast to the D-band, no defects are required for this mode to occur in the Raman spectrum. ${ }^{29}$ At the Raman shift between 0 and $\sim 350 \mathrm{~cm}^{-1}$, the radial breathing modes (RBM) of single-walled CNTs (SWNTs) can be found. This mode can be used to calculate the diameter of the SWNT using the following relation: ${ }^{27}$

$$
\omega_{R B M}=248 / d_{t},
$$

where $\omega_{R B M}$ is the Raman shift and $d_{t}$ is the diameter of the tube. The diameter of the single-walled CNTs grown by our LACVD method is calculated to be in the range of 1.1$1.6 \mathrm{~nm}$. The most abundant diameter was $1.3 \mathrm{~nm}$ which is slightly smaller than the thickness of the original iron layer.

In Fig. 12, the Raman spectra for a number of closedloop controlled experiments are shown. There are two types of experiments. First, the laser saturation irradiance level is increased and the radiation (and thus the temperature) is kept constant. In this case, the radiation was set at $0.4 \mathrm{~V}$ corresponding to $\sim 510^{\circ} \mathrm{C}$. In this figure, it can be seen that increasing the laser saturation level does not improve the quality of the nanotubes. This is shown in more detail in Fig. 13(a). The black dots depict the $\mathrm{I}(\mathrm{D}) / \mathrm{I}(\mathrm{G})$ ratio as a function of laser intensity for the same experimental conditions and are more or less constant which is confirmed by the linear fit. For comparison, two open loop experiments are shown in red dots. There, it is clear that the ratio is much higher for

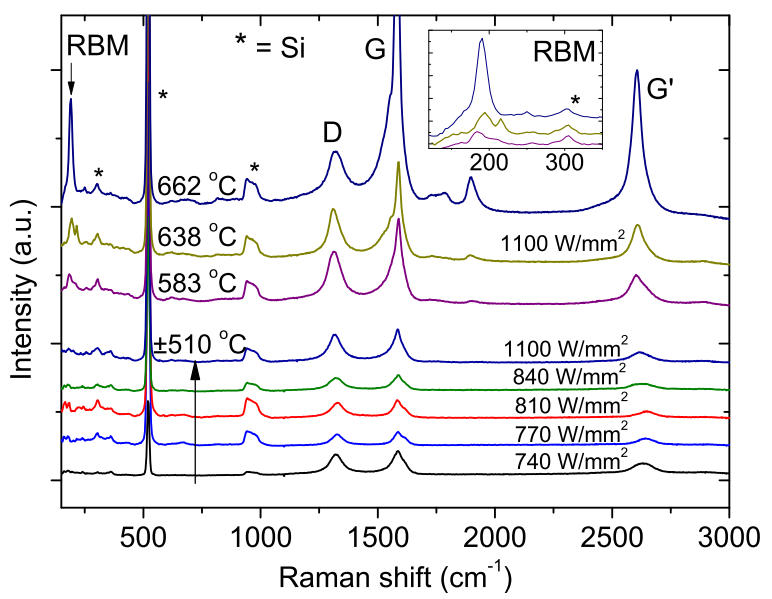

FIG. 12. Raman spectra of CNTs prepared at different laser saturation irradiances and temperatures. All experiments are performed for the duration of $20 \mathrm{~s}$. The curves are shifted in the vertical direction for clarity. The level of irradiance and temperature is indicated next to each spectrum or group of spectra. The inset shows a detailed zoom of the RBM area of the top three spectra. 

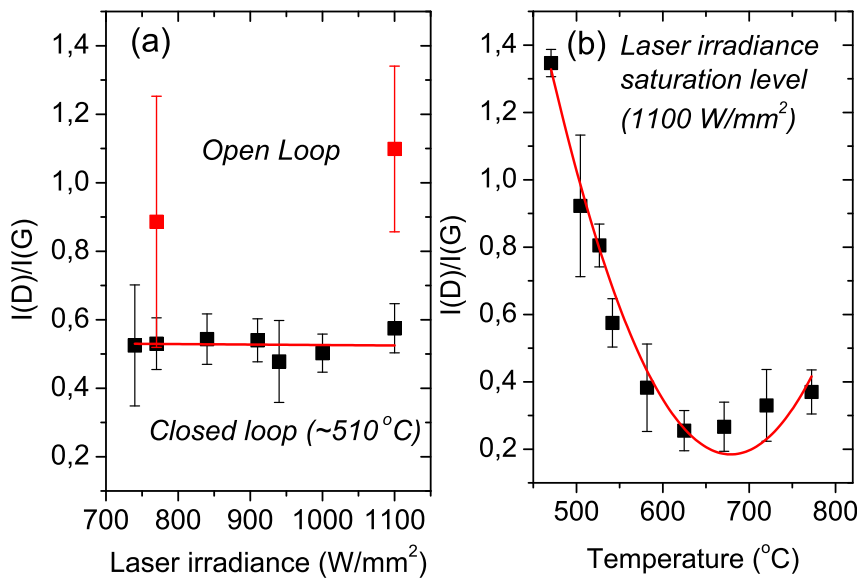

FIG. 13. (a) $\mathrm{I}(\mathrm{D}) / \mathrm{I}(\mathrm{G})$ ratio as a function of laser irradiance. In open-loop, the irradiance represents the set-point of the laser irradiance and in closedloop it represents the laser irradiance saturation level. (b) $\mathrm{I}(\mathrm{D}) / \mathrm{I}(\mathrm{G})$ ratio as a function of temperature for a constant laser irradiance saturation level. Squares and error bars show the mean and standard deviation, respectively, over multiple positions in the center of the laser spot and multiple experiments.

both open-loop experiments. In the case of $1100 \mathrm{~W} / \mathrm{mm}^{2}$, the SEM analysis shows that the substrate was overheated and that a thick amorphous layer was formed. Increasing the reference radiation (i.e., the temperature) for the top three Raman spectra in Fig. 12 increases the temperature at the laser spot yielding higher quality nanotubes. Indeed, the $\mathrm{I}(\mathrm{D}) / \mathrm{I}(\mathrm{G})$ ratio decreases and the presence of the RBM peak demonstrates the presence of SWNTs. The RBM peaks are shown in more detail in the inset. In Fig. 13(b), the I(D)/I(G) ratio is plotted as a function of the process temperature with constant laser saturation irradiance level. The error bars show the standard deviation over multiple experiments and positions at the center of the laser affected zone. The temperature is calculated using the finite element model and the time dependent laser irradiance and reflection as described before. The saturation laser irradiance level was set to the maximum value $\left(1100 \mathrm{~W} / \mathrm{mm}^{2}\right)$ to achieve the highest heating rate. A quadratic fit is added and a minimum is found around $675^{\circ} \mathrm{C}$. This result demonstrates the capability of a temperature-controlled laser assisted CNT growth process. Further, it shows that the CNT quality is a function of the growth temperature in the investigated temperature range if all other parameters are kept constant. Based on Raman spectra, SEM analysis and the non-uniform temperature distribution of the experiments, the grown CNTs are most likely to be a mix of metallic and semi-conducting CNTs as well as multi-walled CNT (MWCNT) and SWCNT.

In Fig. 14, the SEM pictures of a number of those experiments are shown. Figs. 14(a) to 14(c) correspond to the second, fourth and fifth spectra in Figs. 12 and 14(d)14(f) correspond to the top three spectra in Fig. 12. In (a) to (c), the increase of the saturation laser irradiance level seems to affect the length of the CNTs forest. This might be a consequence of the temperature heating rate which influences the efficiency and the nature of the catalyst decomposition. In Figs. 14(d)-14(f), it seems that the amount of CNTs is decreased drastically by increasing temperature. The
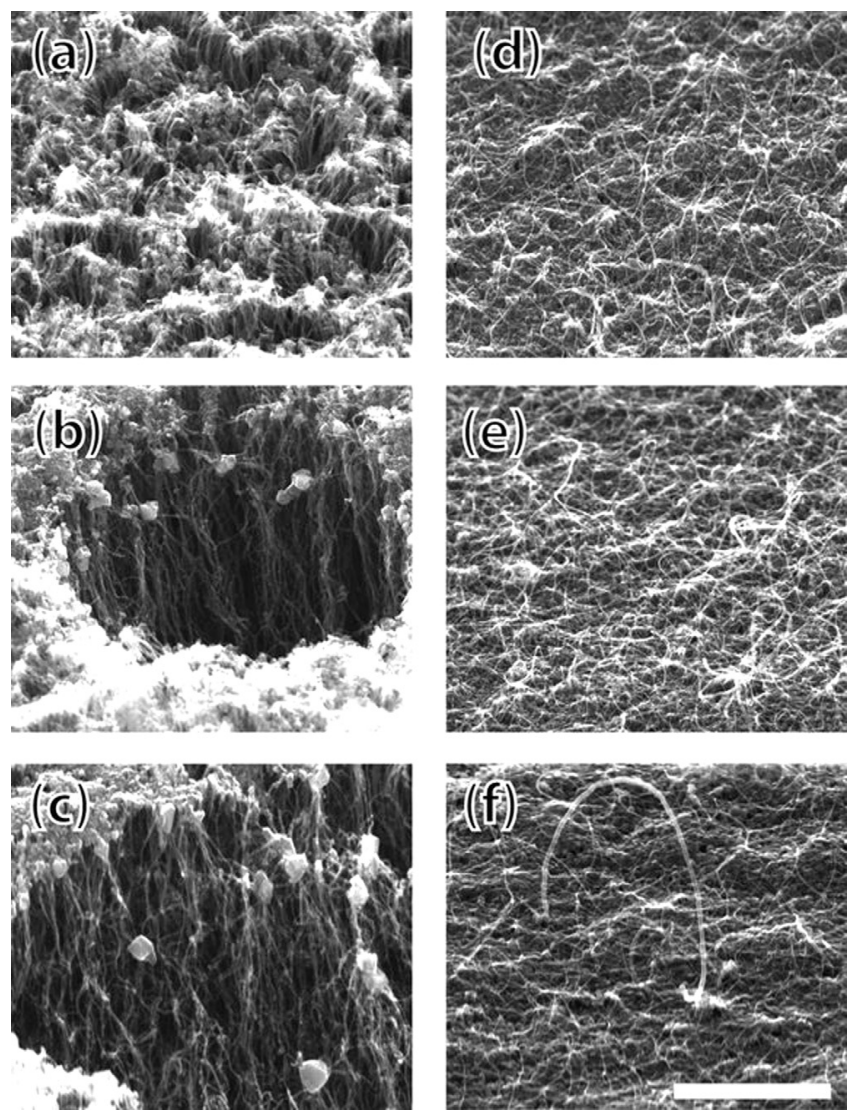

FIG. 14. SEM pictures of the CNT growth for 6 experiments. (a) $\mathrm{I}=770 \mathrm{~W} /$ $\mathrm{mm}^{2}$ and $\mathrm{T}=510^{\circ} \mathrm{C}$. (b) $\mathrm{I}=940 \mathrm{~W} / \mathrm{mm}^{2}$ and $\mathrm{T}=510^{\circ} \mathrm{C}$. (c) $\mathrm{I}=1100 \mathrm{~W} / \mathrm{mm}^{2}$ and $\mathrm{T}=510^{\circ} \mathrm{C}$. (d) $\mathrm{I}=1100 \mathrm{~W} / \mathrm{mm}^{2}$ and $\mathrm{T}=583^{\circ} \mathrm{C}$. (e) $\mathrm{I}=1100 \mathrm{~W} / \mathrm{mm}^{2}$ and $\mathrm{T}=638^{\circ} \mathrm{C}$. (f) $\mathrm{I}=1100 \mathrm{~W} / \mathrm{mm}^{2}$ and $\mathrm{T}=662^{\circ} \mathrm{C}$. The scale bar represents $1 \mu \mathrm{m}$.

increase in temperature results in a few high quality CNTs (low $\mathrm{I}(\mathrm{D}) / \mathrm{I}(\mathrm{G})$ ) while at the same time the growth conditions are changed such that an overall forest growth is absent. This type of growth could be the result of the sensitive process conditions around the laser spot at those high temperatures. The temperature is high enough for SWCNTs to grow but is also closer to thermal decomposition temperature of ethylene and above the wüstite formation temperature of $577^{\circ} \mathrm{C}$. A formation of that oxide can slow down the reduction with hydrogen bringing the growth to a pre-mature stand-still. Although we do not have clear evidence to support it, the crystal structure of the oxide nevertheless resembles that of other iron-oxides.

\section{Catalyst activation control}

Using the information from the reflected laser irradiation as described earlier, we have the possibility to detect the onset of the catalyst layer decomposition, which can be used to optimize the gas feeding schedule. For non-laser CVD, a catalyst preparation step is common prior to the CNT growth. Here, we show that the same preparation step can be done with laser assisted CVD. As a consequence of the fast heating rate due to the low thermal inertia of the system, the catalyst preparation step is considerably shortened.

As a proof-of-concept, we demonstrate the growth of aligned CNT by introducing the flow of ethylene just after 

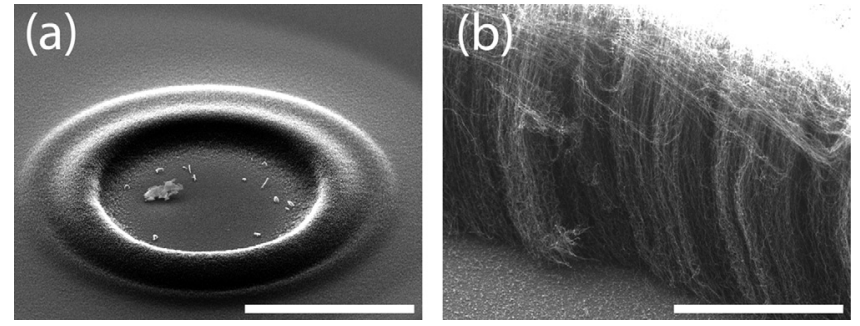

FIG. 15. (a) SEM Image of a donut shaped forest of aligned carbon nanotubes grown by flowing ethylene after the catalyst activation step measured by the reflected laser irradiation. The scale bar represents $200 \mu \mathrm{m}$. (b) SEM picture of a detailed zoom of the edge of the forest after scratching with a knife. The vertically aligned CNTs are visible. The scale bar represents $10 \mu \mathrm{m}$.

the catalyst activation step (detected using our method described earlier). Specifically, the chamber is filled with argon and hydrogen to a pressure of 290 mbar. The temperature set-point value (calculated by the thermal model which also includes a forced convection component for the ethylene) is set to $600^{\circ} \mathrm{C}$. The saturation laser irradiance level is $1100 \mathrm{~W} / \mathrm{mm}^{2}$ and the ethylene is flowed at a rate of 250 $\mathrm{sccm}$. The process time is set to $120 \mathrm{~s}$. The result is shown in Fig. 15. The donut shape suggests excessive temperature in the center of the laser affected zone which suggests further optimization of the process is needed. Nevertheless, this proof of principle demonstrates the controlled sequence of catalyst preparation followed by CNTs growth, both performed with the same laser.

\section{CONCLUSION}

We have developed a closed-loop control process based on the infrared emission from the laser spot for reproducibly growing local CNT forests using a laser assisted CVD method. Finite element modeling, calibrated with experimental data, is used to determine and compare process temperatures. From experiments in a non-growth environment, we have investigated the iron catalyst properties and found that the optimal catalyst reduction and formation occurs when the maximum laser irradiance of $1100 \mathrm{~W} / \mathrm{mm}^{2}$ is used to expose the catalyst layer for 6 seconds to ensure formation of small particles but to avoid the formation of oxide crystals. We found a unique method to detect the onset of the catalyst layer monitoring the reflected laser irradiance. Increasing the growth temperature yields the same trend as for conventional CVD growth with respect to the quality of the CNTs. A minimum of the $\mathrm{I}(\mathrm{D}) / \mathrm{I}(\mathrm{G})$ ratio in the Raman spectra is found around $675^{\circ} \mathrm{C}$. The amount and alignment of the CNTs, however, change rapidly for high temperatures suggesting iron-oxide formation and thermal decomposition of ethylene at high temperatures. Using the information from the reflected laser radiation, we have given a proof-of-concept for growing local CNT structures by introducing the carbon-containing gas triggered by completion of catalyst reduction and formation. This resulted in aligned CNT growth. With this novel laser assisted CVD method, we have successfully demonstrated that growing local CNT structures is possible. This opens up possibilities for different applications such as interconnects in flexible electronics where non-homogenous substrates require a stable temperature controlled growth of these CNT structures.

\section{ACKNOWLEDGMENTS}

The work presented has been financially supported through a research grant offered by a 3TU contract with TNO Holst Centre.

${ }^{1}$ R. H. Baughman, A. A. Zakhidov, and W. A. De Heer, Science 297, 787 (2002).

${ }^{2}$ S. J. Tans, A. R. M. Verschueren, and C. Dekker, Nature 393, 49 (1998).

${ }^{3}$ R. Martel, T. Schmidt, H. R. Shea, T. Hertel, and P. Avouris, Appl. Phys. Lett. 73, 2447 (1998).

${ }^{4}$ W. B. Choi, D. S. Chung, J. H. Kang, H. Y. Kim, Y. W. Jin, I. T. Han, Y. H. Lee, J. E. Jung, N. S. Lee, G. S. Park, and J. M. Kim, Appl. Phys. Lett. 75, 3129 (1999).

${ }^{5}$ J. Kong, N. R. Franklin, C. Zhou, M. G. Chapline, S. Peng, K. Cho, and H. Dai, Science 287, 622 (2000).

${ }^{6}$ T. Y. Tsai, C. Y. Lee, N. H. Tai, and W. H. Tuan, Appl. Phys. Lett. 95, 013107 (2009).

${ }^{7}$ M. Mahjouri-Samani, Y. S. Zhou, W. Xiong, Y. Gao, M. Mitchell, L. Jiang, and Y. F. Lu, Nanotechnology 21, 395601 (2010).

${ }^{8}$ F. Rohmund, R.-E. Morjan, G. Ledoux, F. Huisken, and R. Alexandrescu, J. Vac. Sci. Technol. B 20, 802 (2002).

${ }^{9}$ S. N. Bondi, W. J. Lackey, R. W. Johnson, X. Wang, and Z. L. Wang, Carbon 44, 1393 (2006).

${ }^{10}$ J. B. Park, S. H. Jeong, M. S. Jeong, S. C. Lim, I. H. Lee, and Y. H. Lee, Nanotechnology 20, 185604 (2009).

${ }^{11}$ Z. Liu, D. Styers-Barnett, A. Puretzky, C. Rouleau, D. Yuan, I. Ivanov, K. Xiao, J. Liu, and D. Geohegan, Appl. Phys. A: Mater. Sci. Process. 93, 987 (2008).

${ }^{12}$ A.-C. Dupuis, Prog. Mater. Sci. 50, 929 (2005).

${ }^{13}$ G. D. Nessim, A. J. Hart, J. S. Kim, D. Acquaviva, J. Oh, C. D. Morgan, M. Seita, J. S. Leib, and C. V. Thompson, Nano Lett. 8, 3587 (2008).

${ }^{14}$ S. Linderoth, S. Mørup, and M. D. Bentzon, J. Mater. Sci. 30, 3142 (1995).

${ }^{15}$ A. Moisala, A. G. Nasibulin, and E. I. Kauppinen, J. Phys.: Condens. Matter 15, 3011 (2003).

${ }^{16}$ M. Haluska, Y. Bellouard, Y. van de Burgt, and A. Dietzel, Nanotechnology 21, 7 (2010).

${ }^{17}$ C. L. Yaws, Matheson Gas Data Book, 7th ed. (McGraw-Hill, 2001).

${ }^{18}$ S. W. Churchill and H. H. S. Chu, Int. J. Heat Mass Transfer 18, 1323 (1975).

${ }^{19}$ O. A. Nerushev, S. Dittmar, R.-E. Morjan, F. Rohmund, and E. E. B. Campbell, J. Appl. Phys. 93, 4185 (2003).

${ }^{20}$ Y. Y. Wei, G. Eres, V. I. Merkulov, and D. H. Lowndes, Appl. Phys. Lett. 78, 1394 (2001).

${ }^{21}$ M. V. C. Sastri, R. P. Viswanath, and B. Viswanathan, Int. J. Hydrogen Energy 7, 951 (1982).

${ }^{22}$ A. Pineau, N. Kanari, and I. Gaballah, Thermochim. Acta 447, 89 (2006).

${ }^{23}$ C. Mattevi, C. T. Wirth, S. Hofmann, R. Blume, M. Cantoro, C. Ducati, C. Cepek, A. Knop-Gericke, S. Milne, C. Castellarin-Cudia, S. Dolafi, A. Goldoni, R. Schloegl, and J. Robertson, J. Phys. Chem. C 112, 12207 (2008).

${ }^{24}$ P. B. Amama, C. L. Pint, S. M. Kim, L. McJilton, K. G. Eyink, E. A. Stach, R. H. Hauge, and B. Maruyama, ACS Nano 4, 895 (2010).

${ }^{25}$ H. Sato, Y. Hori, K. Hata, K. Seko, H. Nakahara, and Y. Saito, J. Appl. Phys. 100, 104321 (2006).

${ }^{26}$ M. S. Dresselhaus and P. C. Eklund, Adv. Phys. 49, 705 (2000).

${ }^{27}$ A. Jorio, R. Saito, J. H. Hafner, C. M. Lieber, M. Hunter, T. McClure, G. Dresselhaus, and M. S. Dresselhaus, Phys. Rev. Lett. 86, 1118 (2001).

${ }^{28}$ M. S. Dresselhaus, G. Dresselhaus, R. Saito, and A. Jorio, Phys. Rep. 409, 47 (2005).

${ }^{29}$ R. Saito, A. Jorio, A. G. S. Filho, G. Dresselhaus, M. S. Dresselhaus, A. Grüneis, L. G. Cançado, and M. A. Pimenta, Jpn. J. Appl. Phys., Part 1 41, 4878 (2002). 